\title{
Front Matter: Volume 10598
}

, "Front Matter: Volume 10598," Proc. SPIE 10598, Sensors and Smart Structures Technologies for Civil, Mechanical, and Aerospace Systems 2018, 1059801 (26 June 2018); doi: 10.1117/12.2324924

Event: SPIE Smart Structures and Materials + Nondestructive Evaluation and SPIE. Health Monitoring, 2018, Denver, Colorado, United States 


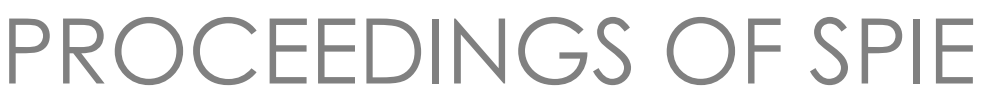

\title{
Sensors and Smart Structures Technologies for Civil, Mechanical, and Aerospace Systems 2018
}

\author{
Hoon Sohn \\ Jerome P. Lynch \\ Kon-Well Wang \\ Editors
}

\author{
5-8 March 2018 \\ Denver, Colorado, United States
}

Sponsored by

SPIE

Cosponsored by

Oz Optics, Ltd. (United States)

Polytec, Inc. (United States)

Cooperating Organizations

Jet Propulsion Laboratory (United States)

Colorado Photonics Industry Association (United States)

Published by

SPIE

\author{
Volume 10598 \\ Part One of Two Parts
}


The papers in this volume were part of the technical conference cited on the cover and title page. Papers were selected and subject to review by the editors and conference program committee. Some conference presentations may not be available for publication. Additional papers and presentation recordings may be available online in the SPIE Digital Library at SPIEDigitalLibrary.org.

The papers reflect the work and thoughts of the authors and are published herein as submitted. The publisher is not responsible for the validity of the information or for any outcomes resulting from reliance thereon.

Please use the following format to cite material from these proceedings:

Author(s), "Title of Paper," in Sensors and Smart Structures Technologies for Civil, Mechanical, and Aerospace Systems 2018, edited by Hoon Sohn, Jerome P. Lynch, Kon-Well Wang, Proceedings of SPIE Vol. 10598 (SPIE, Bellingham, WA, 2018) Seven-digit Article CID Number.

ISSN: 0277-786X

ISSN: 1996-756X (electronic)

ISBN: 9781510616929

ISBN: 9781510616936 (electronic)

Published by

SPIE

P.O. Box 10, Bellingham, Washington 98227-0010 USA

Telephone +1 3606763290 (Pacific Time) · Fax +1 3606471445

SPIE.org

Copyright @ 2018, Society of Photo-Optical Instrumentation Engineers.

Copying of material in this book for internal or personal use, or for the internal or personal use of specific clients, beyond the fair use provisions granted by the U.S. Copyright Law is authorized by SPIE subject to payment of copying fees. The Transactional Reporting Service base fee for this volume is $\$ 18.00$ per article (or portion thereof), which should be paid directly to the Copyright Clearance Center (CCC), 222 Rosewood Drive, Danvers, MA 01923. Payment may also be made electronically through CCC Online at copyright.com. Other copying for republication, resale, advertising or promotion, or any form of systematic or multiple reproduction of any material in this book is prohibited except with permission in writing from the publisher. The CCC fee code is 0277$786 \mathrm{X} / 18 / \$ 18.00$.

Printed in the United States of America.

Publication of record for individual papers is online in the SPIE Digital Library.

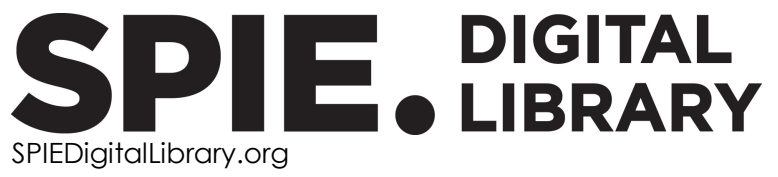

Paper Numbering: Proceedings of SPIE follow an e-First publication model. A unique citation identifier (CID) number is assigned to each article at the time of publication. Utilization of CIDs allows articles to be fully citable as soon as they are published online, and connects the same identifier to all online and print versions of the publication. SPIE uses a seven-digit CID article numbering system structured as follows:

- The first five digits correspond to the SPIE volume number.

- The last two digits indicate publication order within the volume using a Base 36 numbering system employing both numerals and letters. These two-number sets start with $00,01,02,03,04$, 05, 06, 07, 08, 09, OA, OB ... 0Z, followed by 10-1Z, 20-2Z, etc. The CID Number appears on each page of the manuscript. 


\title{
Contents
}

\author{
xiii Authors \\ xvii Conference Committee
}

\section{Part One}

UAV FOR STRUCTURAL INSPECTION

1059802 Challenging issues and solutions of bridge inspection technology using unmanned aerial vehicles [10598-1]

1059804 Damage detection with an autonomous UAV using deep learning [10598-4]

\section{ADVANCED COMPOSITE TECHNOLOGIES}

1059805 Predicting failure from conductivity changes in piezoresistive nanocomposites [10598-5]

1059808 Spatial strain measurements using a strain-sensing grid patterned from nanocomposite films [10598-8]

$1059809 \quad$ Numerical and experimental investigation of matrix effect on sensing behavior of piezoresistive hybrid nanocomposites [10598-9]

\section{HEALTH ASSESSMENT OF COMPOSITE STRUCTURES}

10598 OB Study of CFRP adhesive bonds influenced by manufacturing-related contaminations [10598-10]

10598 OC Study of CFRP adhesive bonds influenced by factors encountered during aircraft operations [10598-11]

10598 OD Damage severity assessment in composite structures using ultrasonic guided waves with chirp excitation [10598-12]

10598 OF Structural health monitoring of a composite F/A-18 wing section using a sparse piezoelectric transducer array [10598-14]

10598 OG Study of disbond effects in a jointed composite structure under variable ambient temperatures [10598-15] 
$10598 \mathrm{OH} \quad$ A planar array capacitive imaging system for detecting damage in composite structures: a numerical study [10598-16]

10598 Ol Strain monitoring using distributed fiber optic sensors embedded in carbon fiber composites [10598-17]

VISION-BASED STRUCTURAL HEALTH MONITORING I

10598 OJ Video-based crack detection using deep learning and Nave Bayes data fusion [10598-18]

10598 OK Machine learning and digital image processing for non-contact modal parameters identification of structures [10598-19]

10598 OL Vision-based concrete crack detection technique using cascade features [10598-21]

10598 ON Development of a flexible capacitive sensor for concrete structure health monitoring [10598-156]

WIRELESS SENSORS AND APPLICATIONS I

10598 OP Design and validation of a wireless distributed control experimental system on three-layer spring steel structure model [10598-23]

$105980 Q \quad$ Dense capacitive sensor array for monitoring distortion-induced fatigue cracks in steel bridges [10598-24]

10598 OR Multifunctional self-powered hydraulic system sensor node [10598-25]

10598 OS Capacitance-based wireless strain sensor development [10598-26]

VISION-BASED STRUCTURAL HEALTH MONITORING II

10598 OT Deep faster R-CNN-based automated detection and localization of multiple types of damage [10598-27]

10598 OU An image-based feature tracking approach for bolt loosening detection in steel connections [10598-28]

10598 OV Automated fatigue crack identification through motion tracking in a video stream [10598-29]

10598 OW Automated volumetric damage detection and quantification using region-based convolution neural networks and an inexpensive depth camera [10598-30]

iv 
WIRELESS SENSORS AND APPLICATIONS II

10598 OX A methodology for structural health diagnosis and assessment using machine learning with noisy and incomplete data from self-powered wireless sensors [10598-31]

10598 OY A compact, low-cost, real-time interrogation system for dynamic interrogation of microstrip patch antenna sensor [10598-32]

10598 OZ Demand-based wireless smart sensors for earthquake monitoring of civil infrastructure [10598-33]

$1059810 \quad$ Piezoelectric charging and wireless communication [10598-34]

\section{DEEP LEARNING FOR STRUCTURAL HEALTH MONITORING}

1059811 Diagnosis of crack damage on structures based on image processing techniques and R-CNN using unmanned aerial vehicle (UAV) [10598-35]

1059812 Deep learning-based concrete crack detection using hybrid images [10598-36]

1059813 Deep learning-based rapid inspection of concrete structures [10598-37]

1059814 Data-driven structural diagnosis and conditional assessment: from shallow to deep learning [10598-38]

\section{NOVEL SENSING TECHNOLOGIES I}

1059818 Micromechanical broadband infrared sensors based on piezoelectric bending resonators [10598-42]

10598 1B Monolithic linear and angular sensors for real-time low-frequency structural distributed monitoring [10598-45]

10598 1C Thick-film resistors on glass ceramic substrates as smart strain sensing aggregates for SHM [10598-46]

10598 1D A rotational actuator based on the piezoelectric bimorph [10598-47]

10598 1E Saliva biomarker detection using an aptamer-based nanosensor [10598-48]

NOVEL SENSING TECHNOLOGIES II

10598 1F Piezoelectric torsional actuation in $\mathbf{d}_{36}$ shear-mode PMN-PT single crystals [10598-50] 
10598 IG Sensor optimization using an evolutionary strategy for structural health monitoring in high temperature environments [10598-51]

10598 1H Response of long-gauge strain sensors in proximity of force application point [10598-52]

$1059811 \quad$ An approach to manipulate frequency selectivity in Basilar metamembrane based broadband frequency sensors [10598-53]

\section{MACHINE LEARNING FOR STRUCTURAL HEALTH MONITORING}

10598 1 J Automated damage-sensitive feature extraction using unsupervised convolutional neural networks [10598-54]

$105981 \mathrm{~K}$ Crack identification inside on-site steel box girder based on fusion convolutional neural network [10598-55]

$10598 \mathrm{lL} \quad$ Online fatigue crack quantification and prognosis using nonlinear ultrasonic modulation and artificial neural network [10598-56]

$105981 \mathrm{M}$ Automated air-coupled impact echo based non-destructive testing using machine learning [10598-57]

\section{MODELING OF SMART MATERIALS AND SENSOR PERFORMANCE}

10598 iN Sensing and control of flexible hydrodynamic lifting bodies in multiphase flows [10598-59]

1059810 Self-sensing characteristics experiment of modified magneto-rheological rubber bearing [10598-61]

10598 IP Fluid-structure coupled acoustic analysis of vibrating Basilar membrane within the cochlea of inner ears [10598-62]

\section{CIVIL INFRASTRUCTURE MONITORING I}

$105981 Q \quad$ Real-time evaluation research of pedestrian-induced footbridge vibration comfort based on smart mobile device [10598-63]

10598 IR Identification of large-scale systems with noisy data using an iterated cubature unscented Kalman filter [10598-64]

10598 IT Strain monitoring in masonry structures using smart bricks [10598-66] 
$105981 \mathrm{U} \quad$ Control of equipment isolation system using wavelet-based decentralized sliding mode control [10598-67]

10598 IV Damage prognosis of China ancient wooden buildings based on structural health monitoring system [10598-68]

10598 IW Discussion of using SSI-COV, refined FDD and multivariate AR model for operational modal analysis [10598-69]

$105981 \mathrm{X} \quad$ Heterogeneous data fusion for impact force identification in truss structures [10598-70]

10598 1Y Discussion of signal decomposition techniques on feature extraction from structural dynamic response data [10598-71]

1059821 Detection of the onset of delamination in a post-tensioned curved concrete structure using hidden Markov modeling of acoustic emissions [10598-74]

\section{Part Two}

\section{CIVIL INFRASTRUCTURE MONITORING II}

1059823 Telemetry techniques for continuous monitoring of partially submerged large civil infrastructure [10598-76]

1059825 Vibration monitoring of a tall building applying DBF based imaging radar: VirA [10598-78]

$1059826 \quad$ Flood fragility analysis of instream bridges [10598-79]

1059827 Input and state estimation for earthquake-excited building structures using acceleration measurements [10598-80]

1059828 Modal property difference formulations and optimization algorithm comparison towards FE model updating [10598-81]

1059829 Seismic damage assessment of a base-isolated bridge using recursive subspace identification algorithm [10598-82]

\section{FIBER OPTIC SENSORS FOR STRUCTURAL HEALTH MONITORING I}

10598 2A In-pavement fiber Bragg grating sensor for vehicle speed and wheelbase estimation [10598-84]

10598 2D Response of an embedded distributed optical fiber sensor to directed energy and applied strain [10598-87]

10598 2E A distributed optical fiber sensing system for data center thermal monitoring [10598-88] 
10598 2F Research on subsea pipeline scour monitoring using distributed Raman optical sensing technique [10598-89]

\section{NOVEL SENSING TECHNOLOGIES III}

10598 2l Bottom-up crack detection in concrete pavements using in-pavement strain sensors [10598-93]

10598 2J Monitoring solid metal structures with a nervous system embedded with ultrasonic 3D printing [10598-94]

10598 2K Grating based high-frequency ultrasonic sensors [10598-95]

\section{SENSOR DEVELOPMENT AND APPLICATIONS}

$105982 \mathrm{~L} \quad$ Comparison of attitude and heading reference systems using foot mounted MIMU sensor data: basic, Madgwick, and Mahony [10598-96]

10598 2M Real-time in-chip phase noise characterization of digitally controlled swept laser source [10598-97]

\section{NEW TECHNOLOGICAL ADVANCES}

$105982 \mathrm{D}$ Design of smart harvester for capturing energy from human ankle dorsiflexion to reduce user effort [10598-101]

$105982 R \quad$ The effects of damage accumulation in optimizing a piezoelectric energy harvester configuration [10598-102]

$105982 S \quad$ Accoustoelastic MetaWall as industrial noise barriers with simultaneous energy harvesting capibility [10598-103]

$105982 \mathrm{~T} \quad$ Output analysis of swarm of neural oscillators stimulated by earthquake-induced acceleration responses of a structure [10598-104]

$105982 \mathrm{U} \quad$ Smart cements: repairs and sensors for concrete assets [10598-105]

\section{INTEGRATION OF SMART SENSING SYSTEMS}

10598 2W Auto-Gopher-II: a wireline rotary-hammer ultrasonic drill that operates autonomously [10598-107]

$105982 X \quad$ System identification and vibration-based damage detection in a concrete shear wall system [10598-109] 
INTERROGATION OF STRUCTURES II

$105982 Y \quad$ Determination of wave velocity for source location of a granite specimen [10598-110]

$1059822 \quad$ A study on the detection of compressed micro-crack by nonlinear wave modulation technique [10598-111]

1059830 3D printed origami as a realization of analysis-driven morphing structures [10598-112]

$1059831 \quad$ Variability analysis of asphalt mixture beam bending test [10598-113]

$1059832 \quad$ Updating the finite element model for electrical impedance tomography using self-organizing map [10598-114]

\section{FIBER OPTIC SENSORS FOR STRUCTURAL HEALTH MONITORING II}

1059833 Real time corrosion detection of rebar using embeddable fiber optic ultrasound sensor [10598-115]

$1059835 \quad$ Monitoring of soil nailed slope stabilizations using distributed fiber optic sensing [10598-117]

1059836 Pipeline internal corrosion sensor based on fiber optics and permanent magnets [10598-118]

1059837 The development of a fiber Bragg grating based smart washer [10598-119]

$1059838 \quad$ Effect of continuous optical fiber bonding on ultrasonic detection using fiber Bragg grating [10598-120]

1059839 Damage detection at web/flange junction of welded I-section steel beam based on impactoptic technique [10598-121]

10598 3A A three-dimensional sliding and debonding sensor based on triaxial optical fiber Fabry-Perot interferometers [10598-122]

INTERNET OF THINGS SENSOR NETWORK

10598 3B Crack detection in RC structural components using a collaborative data fusion approach based on smart concrete and large-area sensors [10598-123]

10598 3D Road sensor network for smart city application [10598-125]

10598 3E Real-time signal processing for sub-THz range grating-based distributed fiber sensing [10598-126] 
10598 3F Surrogate model for condition assessment of structures using a dense sensor network [10598-127]

$105983 \mathrm{H} \quad$ Sparse sensor networks for active structural health monitoring using highly integrated CMOS transceivers [10598-129]

\section{POSTER SESSION}

$1059831 \quad$ Simulation of strain dependent damping in SMA embedded hybrid beams [10598-130]

10598 3J An optical fiber extrinsic Fabry-Perot interferometer based displacement sensor with centimeter measurement range [10598-131]

10598 3K Comparison of binary and multi-level logic processing for an optical encoder [10598-132]

10598 3L A high-linear sweep laser source to interrogate sub-terahertz range fiber sensors for dynamic strain sensing applications [10598-133]

10598 3M Design of a new inside multi-coils clutch for knee-exoskeleton structure based on Helmholtz phenomenon and magneto-rheological fluid [10598-134]

10598 3N Design of a new exoskeleton based on the combination of two magneto-rheological damper [10598-135]

1059830 Wide-range displacement sensor based on a hollow coaxial cable Fabry-Perot resonator [10598-136]

10598 3P Buckled beam based optical interferometric pressure sensor with low temperature crosssensitivity [10598-137]

$105983 Q \quad$ Numerical study of cornea applanation by using a portable force-displacement sensor for intraocular pressure measurements [10598-138]

10598 3R Design and realization of data loss compensation system based on compressed sensing [10598-139]

10598 3S Design and verification of an indoor wireless UWB positioning system for civil structures [10598-140]

10598 3T Inertial monolithic sensors for low frequency acceleration measurement of spacecrafts and satellites [10598-141]

10598 3V Convolutional neural networks-based crack detection for real concrete surface [10598-143]

$1059842 \quad$ Principle of a test bench for simulation of vehicular braking of 1/4 vehicle [10598-150]

1059844 Optimal sensor placement for continuous optical fiber sensors [10598-152] 
1059846 Integrating 3D scanning within a simulation framework for structural mechanics [10598-154]

1059849 Finite element analysis of RC beam strengthened with FOS embedded carbon fiber sheet [10598-158]

$105984 \mathrm{~A}$ Experimental investigation on the FOS embedded carbon fiber sheet for bridge sensing and reinforcement [10598-159] 
Proc. of SPIE Vol. 10598 1059801-12 Downloaded From: https://www.spiedigitallibrary.org/conference-proceedings-of-spie on 26 Apr 2023
Terms of Use: https://www.spiedigitallibrary.org/terms-of-use 


\section{Authors}

Numbers in the index correspond to the last two digits of the seven-digit citation identifier (CID) article numbering system used in Proceedings of SPIE. The first five digits reflect the volume number. Base 36 numbering is employed for the last two digits and indicates the order of articles within the volume. Numbers start with 00, 01, 02, 03, 04, 05, 06, 07, 08, 09, OA, OB...0Z, followed by 10-12, 20-2Z, etc.

Acernese, F., 3T

Ahamed, Touhid, 26

Ahmed, Riaz U., 11, $2 S$

Alexander, Quincy G., 23

Ali, Rahmat, OL

Almahmoud, Safieh, 36

Alshandah, Mohanad, 21

Al-Tarawneh, Mu'ath, 2A

Amrithanath, Abhishek K., 2K

An, Yun-Kyu, 12

Anandan, Sudharshan, 01

Anees, Muhammad, 09

Badescu, Mircea, 10, 2W

Bagchi, A., $2 X$

Bai, Xian-Xu, 42

Banerjee, Sourav, 11, $2 S$

Bao, Xiaoqi, 10, 18, 2W

Bar-Cohen, Yoseph, 10, 2W

Barone, F., 1B, 3T

Bayrak, Oguzhan, 21

Beckman, Gustavo H., OW

Bennett, Caroline, OQ, OS

Berik, Pelin, IF

Berthe, Laurent, $\mathrm{OB}, \mathrm{OC}$

Bhagat, Nitin, 30

$\mathrm{Bi}$, Kevin, 3H

Biondi, Lorena, $2 \mathrm{U}$

Biswas, Subir, OX

Boustie, Michel, OB, OC

Bradford, Philip, 38

Bridgelall, Raj, 3D

Brune, Kai, OB, OC

Burgueno, Rigoberto, OX

Burnham, Kaleb D., $2 \mathrm{~L}$

Burton, Andrew R., 08

Cai, Mingjing, $2 Q$

Cancelli, Alessandro, 3F

Cao, Xuefeng, 35

Cazzulani, G., 44

Cha, Ae-Ri, 3N

Cha, Young-jin, 04, OL, OT, OW, 1 J, 1M, IR

Chakrabartty, Shantanu, OX

Chandra, A., $2 X$

Chandrashekhara, K., 0 I

Chang, Wei-Yi, $1 F$

Chen, An, 3F

Chen, Deyi, $1 Q$

Chen, Dongdong, 37

Chen, Fu-Chen, 0J
Chen, Jiahui, $1 \mathrm{~K}$

Chen, Jian, 42

Chen, Jun-Da, 29

Chen, Xinjian, 3Q

Chen, Zhen, 2E, 2M, 3L

Cheng, Yu, ON

Chieppi, M., 44

Cho, Soojin, 12, 13

Choi, Jongkwon, 21

Choi, Seung-Bok, 3M, 3N

Christopoulos, Angelos, OB, OC

Ciezki, John G., 3K

Cinquemani, S., 3l

Collins, William, OQ, OS

Colombo, A., 44

Cunefare, Kenneth A., OR

Dafydd, Ifan, OD

D'Alessandro, Antonella, 1T, 3B

Das, Saptarshi, OX

Denslow, Kayte, 2J

Dhaliwal, Gurjot S., 01

Dizaji, Mehrdad S., 46

Do, Xuan Phu, 3M, 3N

Dong, Xinjun, 28

Downey, Austin, 1T, 3B, 3F

Du, Cong, 33

Du, Xiaosong, 3F

Du, Yang, 01

Duan, Jennifer, 26

Durscher, Ryan, J., 30

Ebrahimkhanlou, Arvin, 21

Ecault, Romain, OB, OC

Epp, Tyler, 1M

Fang, Houfei, 1D

Fang, Huazhen, 27

Fiborek, Piotr, OG

Frez, Clifford F., 18

Fu, Y., $0 Z$

Fuchi, Kazuko, 30

Gbaguidi, Audrey, 09

Ghorbani, Esmaeil, 1R

Gillman, Andrew S., 30

Giordano, G., 1B, 3T

Glisic, Branko, 1H

Guan, Xinchun, 1C

Gupta, Sumit, $\mathrm{OH}$

Hackney, Drew, 38

Haghdoust, $P$, , 31

Hamilton, Andrea, $2 \mathrm{U}$ 
Hanif, Asad, ON

Harley, Joel B., 3H

Harris, Devin K., 46

Harwood, Casey M., $1 \mathrm{~N}$

Hassan, H., 05

He, Haibo, 2E

Hefferman, Gerald, 2E, 2M, 3E, 3L

Hirschmüller, Sebastian, 35

Hoang, T., OZ

Hong, Jung-Wuk, $2 Z$

Hongu, Jyunichi, 2T

Hou, Yangaing, 1D

Hrynyk, Trevor D., 21

Hsueh, Wen, IW, IY

Huang, Haiying, $O Y$

Huang, Jie, OI, 3A, 3J, 3O, 3P

Huang, Shieh-Kung, $1 \mathrm{U}$

Huang, Ying, 1G, 2A, 2I, 3D

Huo, Linsheng, 37

Iba, Daisuke, 2T

Iwaki, Hideaki, 25

Jackson, Shannon, 10, 2W

Jahanshahi, Mohammad R., OJ

Jang, Keun-Young, 12

Jenkins, Brian, 2D

Jeong, Jong-Hyun, $0 Q$, OS

Jiang, Shao-Fei, IV

Jiang, Shuidong, ID

Jiang, Xiaoning, $1 \mathrm{~F}$

Jiménez, Antonio R., 2L

Jin, Suyeong, $2 Z$

Jo, Hongki, OQ, OS, 1X, 26

Jothibasu, Sasi, 0 I

Joyce, Peter, 2D

Jung, Hyung-Jo, 02, 11

Jung, Kyusan, 49, 4A

Kan, Ren, 3E

Kang, Dongho, 04

Kanterakis, George, OB, OC

Kaur, Amardeep, 0

Kautzman, Mathew, 2D

Kim, Byeongcheol, 49, 4A

Kim, Byunghyun, 12, 13

Kim, Daewon, 09

Kim, Daniel, 2W

Kim, Gi-Woo, 1P

Kim, Hyunwoo, $2 Y$

Kim, In-Ho, 02, 11

Kim, Jeong Hwan, 1P

Kim, Min-Koan, $2 Y$

Kim, Yooil, 1P

Kitsianos, Konstantinos, OB, OC

Kjolsing, Eric J., 2R

Klein, Jordan D., 23

Kong, Xiangxiong, OQ, OS, OU, OV

Koulalis, llias, OB, OC

Kralovec, Christoph, 32

Krishnaswamy, Sridhar, 2K

Kudela, Pawel, OG

Kuo, Tsai-Jung, IW
Laflamme, Simon, $0 Q, 0 S, 1 T, 3 B, 3 F$

Larimer, Curtis, 2J

Lecis, N., 31

Lee, Hyeong Jae, 10

Lee, Jin-Hwan, 02, 11

Lee, Jong-Won, $2 Y$

Lee, Sang Eon, $2 Z$

Lee, Yein, 13

Lei, Ting, 35

Leifsson, Leifur, 3F

Leite Cavalcanti, Welchy, OB, OC

Le Tran Huy, Thang, 3M

Li, Chuan-Fu, 1Y, 29

Li, Hongnan, 37

Li, Hui, 1C, 1K

Li, Jian, OQ, OS, OU, OV, 27

Li, LUYu, OP

Li, Mingli, 14

Li, Ni-Lei, IV

Li, Rui, 10

Li, Shengyuan, 3V

Li, Yang, 42

$\mathrm{Li}$, Zongjin, ON

Liao, Wei-Hsin, $2 Q$

Liao, Zeng-Cheng, 42

Lienhart, Werner, 35

Lim, Hyung Jin, 1L, $2 Z$

Lin, Zhibin, 14

Liu, Lei, 1D

Liv, Lin, 10

Liv, Wanqiu, 31

Liu, Xu, 31

Liu, Zhiqiang, OP

Lo Conte, A., 31

Loh, Chin-Hsiung, 1U, 1W, 1Y, 29

Loh, Kenneth J., $\mathrm{OH}$

Lokekere Gopal, Dharshan, OL

Lu, Pan, 2I, 3D

Lu, Wen, 31

Ludwig, Simone A., 1G, 2L

Lynch, Jerome P., 08

Mai Bui Quoc, Long, 3N

Malinowski, Pawel H., OB, OC

Mandal, Soumayjit, $3 \mathrm{H}$

Marek, Rachel, $1 \mathrm{H}$

Marte, Roman, 35

McCullum, Jacob R., OF

Mechitov, K., OZ

Mellerowicz, Bolek, 2W

Meoni, Andrea, IT

Metz, Brandon, 2W

Mir, Fariha, 2S

Monsberger, Christoph, 35

Moriwaki, Ichiro, 2T

Namilae, Sirish, 09

Nazarian, Sheyda, 1E

Nelson, Charles, 2D

Netchaev, Anton, 23

Nguyen, Quoc Hung, 3N

Nie, Baoqing, $3 Q$ 
Niu, Li, 39

Nohmi, Akira, 25

Nohmi, Hitoshi, 25

Oh, Tae-Min, $2 Y$

Ostachowicz, Wieslaw M., OB, OC, OG

Ou, Jinping, $1 \mathrm{C}$

Owusu Twumasi, Jones, 33

Ozdemir, Tayfun, 3H

Pan, Hong, 14

Pankonien, Alexander M., 30

Park, Jongsup, 49

Park, Joonseok, 49, 4A

Park, K. T., OK

Park, Kitae, 49, 4A

Park, Sang-Ki, $2 Y$

Paulsen, Gale L., 2W

Pawloski, Martin B., 3H

Pei, Shuyi, 2E

Pennacchi, P., 44

Perry, Marcus, $2 U$

Peters, Kara, 38

Pines, Darryll J., OF

Polyzois, Dimos, OW

Rais-Zadeh, Mina, 18

Renne, Caitlyn M., 3K

Romano, R., 1B, 3T

Rostron, Paul, 36

Saadatzi, Mohammad Sadegh, 25

Sabamehr, A., 2X

Sagnard, Maxime, OB, OC

Salamone, Salvatore, 21

Saleem, Muhammad M., IX

Salehi, Hadi, OX

Schagerl, Martin, 32

Segur, Damien, OB, OC

Seo, Dongwoo, 4A

Sharif Khodaei, Zahra, OD

Shen, Sheng, IV

Sherrit, Stewart, 10, 18, 2W

Shim, Jaeho, 26

Shima, Sohei, 2T

Shirai, Ikuo, 25

Shiryayev, Oleg, 36

Sikdar, Shirsendu, OG

Simonini, Alan, 2W

Skilskyj, James, OY

Skow, Ellen, OR

Smith, Matthew D., 23

Sohn, Hoon, 1L, $2 Z$

Soltani, A., 2X

Song, Gangbing, 37

Spencer, B. F., Jr., OZ

Stoessel, Rainer, OB, OC

Suh, Gahyun, OT

Sun, Peng, 08

Suter, Jonathan D., 2J

Svecova, Dagmar, 1M

Ta Duc, Huy, 3M

Taher, Sdiq, 27

Tallman, T. N., 05
Tamura, Kazuo, 25

Tang, Bo, 2E

Tang, Qixiang, 33

Tang, Xinyao, $3 \mathrm{H}$

Thurmer, Clayton R., 23

Ting, Ethian, OR

Todd, Michael D., 2R

Tokumura, Hideya, 2T

Tolliver, Denver, 2I

Toothman, Maxwell F., OR

Torbol, M., OK

Touchard, Fabienne, OB, OC

Touma, Pierre A., 2L

Ubertini, Filippo, 1T, 3B, 3F

Vahdati, Nader, 36

Vlachakis, Christos, $2 \mathrm{U}$

Wandowski, Tomasz, OB, OC

Wang, Jie, 3S

Wang, Ming L., 1E

Wang, Minglian, 10

Wang, Xingwei, 33

Wang, Xingyu, 14

Wang, Yang, 28

Wang, Zilong, $1 \mathrm{~J}$

Ward, Jacob C., IN

Watkins, Steve E., Ol, 3K

Wee, Junghyun, 38

Wei, Heming, 2K

Wei, Tao, 2E, 2M, 3E, 3L

Wen, Ming, 1C

Wereley, Norman M., OF

Wu, Ming-Hao, $1 \mathrm{~V}$

Wu, Nan, 33

$X U$, Jian, OS

$X U$, Xingxing, 10

$\mathrm{XU}$, Yang, $1 \mathrm{~K}$

$X U$, Ying, 39

Xue, Ruidan, 3R

Yan, Jin, 3F

Yan, Quansheng, 1Q

Yang, Changping, $\mathrm{OP}$

Yang, Ping'an, 10

Yang, Qing, 2E

Yang, Shuzhen, 46

Yao, Jun, OY

Yao, Ting, $3 \mathrm{Q}$

Yao, Zheyi, 2M, 3E, 3L

Yoo, Byungseok, OF

Yoon, Dalseong, 3M

Yoon, Sung-Sik, 11

Young, Yin LU, $1 \mathrm{~N}$

Yu, I-No, 29

Yu, Tzuyang, 33

Yu, Yan, OP, 3R, 3S

Zacny, Kris, 2W

Zhang, Lei, 31

Zhang, Wenjun, $1 \mathrm{E}$

Zhang, Xinwang, 2F

Zhang, $Y i, 3 R$

Zhang, Ying, $1 \mathrm{~V}$ 
Zhao, Xuefeng, 2F, 3V

Zhao, Yingjun, 32

Zhou, Mengjiao, 10

Zhu, Chen, 3A, 3J, 30, 3P

Zhu, L., OZ

Zu, Hongguang, 39

Proc. of SPIE Vol. 10598 1059801-16

Downloaded From: https://www.spiedigitallibrary.org/conference-proceedings-of-spie on 26 Apr 2023 Terms of Use: https://www.spiedigitallibrary.org/terms-of-use 


\title{
Conference Committee
}

\author{
Symposium Chairs
}

Tribikram Kundu, The University of Arizona (United States)

Gregory W. Reich, Air Force Research Laboratory (United States)

Symposium Co-chairs

Zoubeida Ounaies, The Pennsylvania State University (United States)

Hoon Sohn, KAIST (Korea, Republic of)

Conference Chair

Hoon Sohn, KAIST (Korea, Republic of)

Conference Co-chairs

Jerome P. Lynch, University of Michigan (United States)

Kon-Well Wang, University of Michigan (United States)

Conference Program Committee

Hiroshi Asanuma, Chiba University (Japan)

Chih Chen Chang, Hong Kong University of Science and Technology (Hong Kong, China)

Genda Chen, Missouri University of Science and Technology (United States)

Alison B. Flatau, University of Maryland, College Park (United States)

Branko Glisic, Princeton University (United States)

Faramarz Gordaninejad, University of Nevada, Reno (United States)

Benjamin L. Grisso, Naval Surface Warfare Center Carderock Divison (United States)

Ryan L. Harne, The Ohio State University (United States)

Jung-Wuk Hong, KAIST (Korea, Republic of)

Neil A. Hoult, Queen's University (Canada)

Haiying Huang, The University of Texas at Arlington (United States)

Ying Huang, North Dakota State University (United States)

Mohammad Reza Jahanshahi, Purdue University (United States)

Gi-Woo Kim, Inha University (Korea, Republic of)

Jeong-Tae Kim, Pukyong National University (Korea, Republic of)

Masahiro Kurata, Kyoto University (Japan)

Simon Laflamme, lowa State University (United States)

Hui Li, Harbin Institute of Technology (China) 
Jian Li, The University of Kansas (United States)

Suyi Li, Clemson University (United States)

Wei-Hsin Liao, The Chinese University of Hong Kong (Hong Kong, China)

Chin-Hsiung Loh, National Taiwan University (Taiwan)

Kenneth J. Loh, University of California, San Diego (United States)

Bryan R. Loyola, Sandia National Laboratories (United States)

Theodore E. Matikas, University of loannina (Greece)

Norbert G. Meyendorf, lowa State University (United States)

Akira Mita, Keio University (Japan)

Yiqing Ni, The Hong Kong Polytechnic University (Hong Kong, China)

Hae Young Noh, Carnegie Mellon University (United States)

Irving J. Oppenheim, Carnegie Mellon University (United States)

Wieslaw M. Ostachowicz, The Szewalski Institute of Fluid-Flow Machinery (Poland)

Kara J. Peters, North Carolina State University (United States)

Michael K. Philen, Virginia Polytechnic Institute and State University (United States)

Piervincenzo Rizzo, University of Pittsburgh (United States)

Massimo Ruzzene, Georgia Institute of Technology (United States)

Donghyeon Ryu, New Mexico Institute of Mining and Technology (United States)

Liming W. Salvino, Office of Naval Research Global (United States)

Fabio Semperlotti, Purdue University (United States)

Wei Song, The University of Alabama (United States)

Wieslaw J. Staszewski, AGH University of Science and Technology (Poland)

R. Andrew Swartz, Michigan Technological University (United States)

Tyler N. Tallman, Purdue University (United States)

Marco Torbol, Ulsan National Institute of Science and Technology

(Korea, Republic of)

Ming L. Wang, Northeastern University (United States)

Xingwei Wang, University of Massachusetts Lowell (United States)

Yang Wang, Georgia Institute of Technology (United States)

Rosalind M. Wynne, Villanova University (United States)

Fuh-Gwo Yuan, North Carolina State University (United States)

Chung-Bang Yun, Zhejiang University (China)

Daniele Zonta, Università degli Studi di Trento (Italy)

\section{Session Chairs}

$1 \quad$ UAV for Structural Inspection

Hyung-Jo Jung, KAIST (Korea, Republic of)

Steve E. Watkins, Missouri University of Science and Technology (United States) 
2 Advanced Composite Technologies

Donghyeon Ryu, New Mexico Institute of Mining and Technology (United States)

Kenneth J. Loh, University of California, San Diego (United States)

3 Health Assessment of Composite Structures

Wieslaw M. Ostachowicz, The Szewalski Institute of Fluid-Flow Machinery (Poland)

Tyler N. Tallman, Purdue University (United States)

4A Vision-Based Structural Health Monitoring I

Mohammad Reza Jahanshahi, Purdue University (United States)

Marco Torbol, Ulsan National Institute of Science and Technology (Korea, Republic of)

4B Wireless Sensors and Applications I

Hongki Jo, The University of Arizona (United States)

Jian Li, The University of Kansas (United States)

5A Vision-Based Structural Health Monitoring II

Young-Jin Cha, University of Manitoba (Canada)

Jian Li, The University of Kansas (United States)

5B Wireless Sensors and Applications II

Kon-Well Wang, University of Michigan (United States)

Haiying Huang, The University of Texas at Arlington (United States)

6A Deep Learning for Structural Health Monitoring

Yun-Kyu An, Sejong University (Korea, Republic of)

Soojin Cho, Ulsan National Institute of Science and Technology

(Korea, Republic of)

6B Novel Sensing Technologies I

Hui Li, Harbin Institute of Technology (China)

Ming L. Wang, Northeastern University (United States)

7A Novel Sensing Technologies II

Branko Glisic, Princeton University (United States)

Kara J. Peters, The National Science Foundation (United States)

7B Machine Learning for Structural Health Monitoring

Young-Jin Cha, University of Manitoba (Canada)

Yiqing Ni, The Hong Kong Polytechnic University (Hong Kong, China)

8A Modeling of Smart Materials and Sensor Performance

Yooil Kim, INHA University (Korea, Republic of)

Yang Wang, Georgia Institute of Technology (United States) 
8B Civil Infrastructure Monitoring I

Simon Laflamme, lowa State University (United States)

Jerome P. Lynch, University of Michigan (United States)

9A Interrogation of Structures I

Peng Sun, National Taiwan University (Taiwan)

Fabio Semperlotti, Purdue University (United States)

9B Civil Infrastructure Monitoring II

Jian Li, The University of Kansas (United States)

Shieh-Kung Huang, National Taiwan University (Taiwan)

10A Fiber Optic Sensors for Structural Health Monitoring I

Ying Huang, North Dakota State University (United States)

R. Brian Jenkins, U.S. Naval Academy (United States)

10B Novel Sensing Technologies III

Sridhar Krishnaswamy, Northwestern University (United States)

Genda Chen, Missouri University of Science and Technology (United States)

11 A Sensor Development and Applications

Hae-Bum A. Yun, University of Central Florida (United States)

11 B New Technological Advances

Wei-Hsin Liao, The Chinese University of Hong Kong (Hong Kong, China)

12A Integration of Smart Sensing Systems

Gi-Woo Kim, INHA University (Korea, Republic of)

Ying Huang, North Dakota State University (United States)

12B Interrogation of Structures II

Jung-Wuk Hong, KAIST (Korea, Republic of)

Wanqiu Liu, Dalian University of Technology (China)

13A Fiber Optic Sensors for Structural Health Monitoring II

Xingwei Wang, University of Massachusetts Lowell (United States)

Genda Chen, Missouri University of Science and Technology (United States)

13B Internet of Things Sensor Network

Dryver R. Huston, The University of Vermont (United States)

Filippo Ubertino, Università delgi studi di Perugi (Italy) 